\title{
Nonlinear Optical Properties of Novel Mono-O-Hydroxy Bidentate Schiff Base: Quantum Chemical Calculations
}

\author{
N. S. Labidi ${ }^{1,2}$ \\ ${ }^{1}$ Department of Chemistry, Faculty of Sciences, University of the Sciences and Technology of Oran (U.S.T.O.MB), \\ BP 1505 El-M'naouer, 31000 Oran, Algeria \\ ${ }^{2}$ Centre Universitaire de Tamanrasset, 11000 Tamanrasset, Algeria
}

Correspondence should be addressed to N. S. Labidi; labidi19722004@yahoo.fr

Received 5 September 2013; Accepted 17 October 2013

Academic Editor: Tao Zhang

Copyright (C) 2013 N. S. Labidi. This is an open access article distributed under the Creative Commons Attribution License, which permits unrestricted use, distribution, and reproduction in any medium, provided the original work is properly cited.

\begin{abstract}
The semiempirical AM1 SCF method is used to study the first static hyperpolarizabilities $\beta$ of some novel mono-O-Hydroxy bidentate Schiff base in which electron donating (D) and electron accepting (A) groups were introduced on either side of the Schiff base ring system. Geometries of all molecules were optimized at the semiempirical AM1. The first static hyperpolarizabilities of these molecules were calculated using Hyperchem package. To understand this phenomenon in the context of molecular orbital picture, we examined the molecular HOMO and molecular LUMO generated via Hyperchem. The study reveals that the monoO-Hydroxy bidentate Schiff bases have large $\beta$ values and hence in general may have potential applications in the development of nonlinear optical materials.
\end{abstract}

\section{Introduction}

An intense research activity is currently associated with the synthesis and development of molecule-based secondorder nonlinear optical (NLO) materials, involving organic chromophore and metal complexes [1-3]. The Schiff base compounds have been under investigation for several years because of their potential application to optical communications and because many of them have NLO behaviour $[4,5]$. The design of efficient organic materials for the nonlinear effect is based on molecular units containing highly delocalized $\Pi$-electron moieties and extra electron donor and electron acceptor groups on opposite sides of the molecule at appropriate positions on the ring to enhance the conjugation. The effect of electron withdrawing and electron attracting substituents on the first hyperpolarizability $\beta$ of conjugated systems has received a great deal of attention in recent years $[6,7]$. It was shown that the type of substituent plays a major role in charge transfer through the molecule and therefore in nonlinear properties [8]. Prasad and Williams [9] explained that the certain classes of organic materials exhibit extremely larger NLO and electrooptic effect. The design of most efficient organic materials for the nonlinear effect is based on molecular units containing highly delocalized pi-electron moieties and extra electron donor (D) and electron acceptor (A) groups on opposite sides of the molecule at appropriate positions on the ring to enhance the conjugation. The pielectron cloud movement from donor to acceptor makes the molecule to be highly polarized. The chromophore design was mainly done by synthetic explorations which are time consuming and costly process. At the same time, measurement of the molecular nonlinear optical coefficients $(\beta)$ requires the use of the well-known electric-field-induced second harmonic (EFISH) experiment, in which only the vector component of $\beta_{\text {tot }}$ parallel to the molecule's ground state dipole moment can be determined [10]. To avoid these problems, the development of quantum procedures for the science of chromophore design and proprieties calculation has taken a different route especially due to the birth of quantum chemistry packages [11]. One of the best computer semiempirical program packages is Hyperchem 7.0 (molecular modelling system) [12]. This package can be used to study the electronic structure and energy of ground and excited states of atoms, molecules, ions, first hyperpolarizability, second hyperpolarizability, and so forth. 


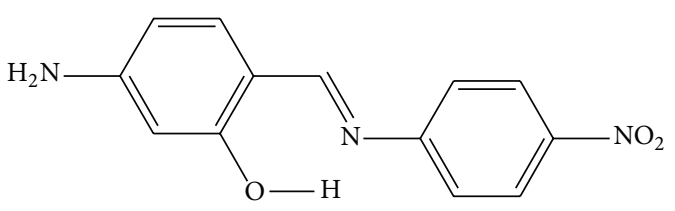

2-((E)-(4-nitrophenylimino)methyl)-5-aminophenol

(1)

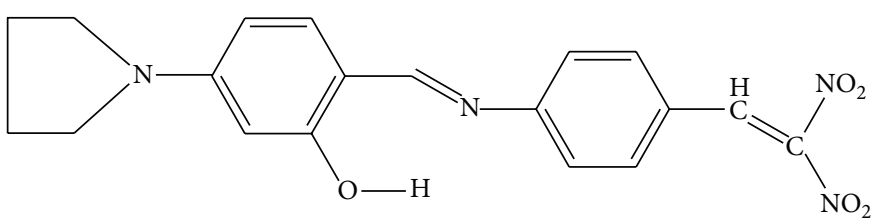

2-((E)-(4-(2,2-dinitrovinyl)phenylimino)methyl)-5-(pyrrolidin-1-yl)phenol

(6)

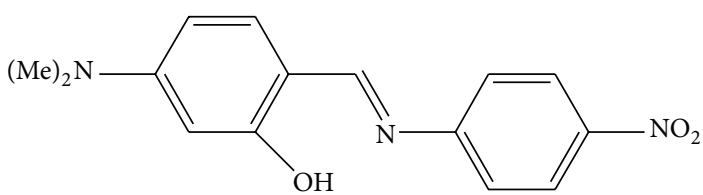

2-((E)-(4-nitrophenylimino)methyl)-5-(dimethylamino)phenol

(2)

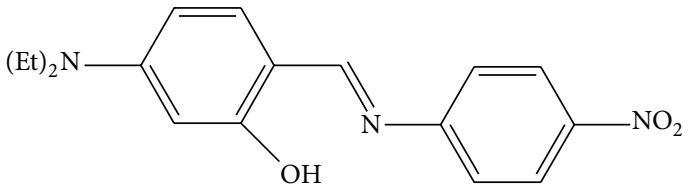

2-((E)-(4-nitrophenylimino)methyl)-5-(dimethylamino)phenol

(3)

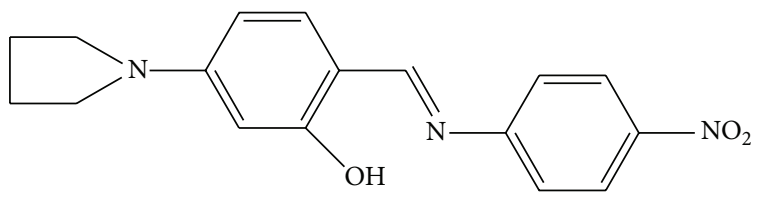

2-((E)-(4-nitrophenylimino)methyl)-5-(pyrrolidin-1-yl)phenol

(4)<smiles>O=[N+]([O-])c1ccc(/N=C/c2ccc(C=C(N3CCCC3)N3CCCC3)cc2O)cc1</smiles>

2-((E)-(4-nitrophenylimino)methyl)-5-(2,2-di(pyrrolidin-1-yl)vinyl)phenol

(5)

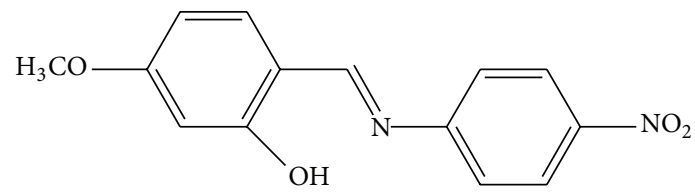

2-((E)-(4-nitrophenylimino)methyl)-5-methoxyphenol

(7)

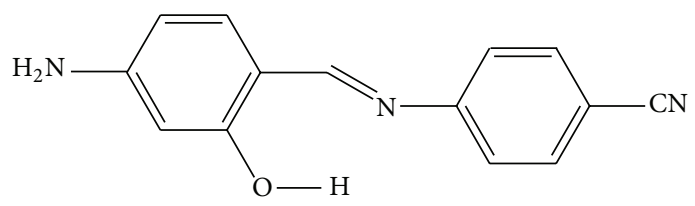

(E)-4-(4-amino-2-hydroxybenzylideneamino)benzonitrile

(8)

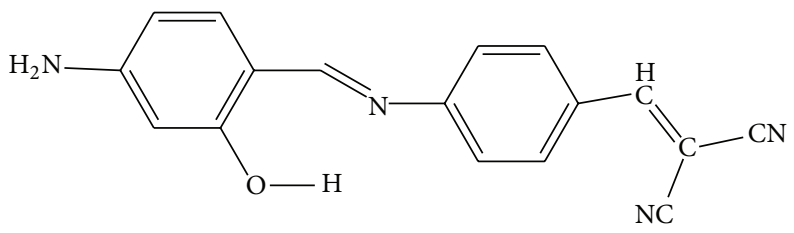

5-amino-2-((E)-(phenylimino)methyl)phenol-2-ethylidenemalononitrile

(9)

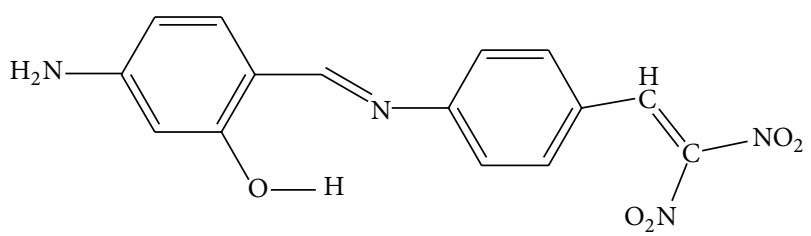

2-((E)-(4-(2,2-dinitrovinyl)phenylimino)methyl)-5-aminophenol

(10)

FIGURE 1: The designed novel mono-O-Hydroxy Schiff base ligands.

Our objective is to design a range of novel asymmetric mono-O-Hydroxy Schiff base compounds as model to evaluate the electronic properties and NLO responses. The approach is based on the concept of charge transfer (CT) between donor and acceptor through the molecules. In this research work, first hyperpolarizabilities $(\beta)$ are calculated using semiempirical AM1 method using Hyperchem program package.

\section{Computational Procedures}

All hyperpolarizability calculations of the O-Hydroxy bidentate Schiff bases (Figure 1) were performed using Hyperchem software [12], Intel Pentium P4 2002 XP with the Windows XP operating system. The AM1 Hamiltonian calculation was used for geometric optimization of the molecules and to calculate parameters like bond length, bond angle, core-core 
attraction, heat of formation, ionisation potential dipole moment, HOMO-LUMO gap, and Mulliken population analysis of the outermost MO for mono-O-Hydroxy Schiff base ligands 1-10.

\section{Second-Order Nonlinearities}

The existence of NLO phenomena is represented at the microscopic level as Taylor's expansion of the relationship between the spatial components of the induced dipole moment $\mu_{i}$ and the components of the electronic field $E_{j}$ that create it $[13,14]$, as shown in

$$
\mu_{i}=\mu_{i}^{0}+\sum \alpha_{i j} E_{j}+\frac{1}{2} \sum \beta_{i j k} E_{j} E_{k}+\frac{1}{6} \sum \gamma_{i j k} E_{j} E_{k} E_{l}+\cdots,
$$

where $\alpha_{i j}=\left(\partial \mu_{i} / \partial E_{j}\right)_{E 0}, \beta_{i j k}=\left(\partial \mu i / \partial E_{j} \partial E_{k}\right)_{E 0}$, and $\gamma_{i j k l}=$ $\left(\partial \mu_{i} / \partial E_{j} \partial E_{k} \partial E_{k} \partial E_{l}\right)_{E 0}$. These tensors can also be developed from Taylor's expansion of the Stark energy, as shown in

$$
\begin{aligned}
U(E)= & U(0)-\sum \mu_{i} E_{i}-\frac{1}{2} \sum \alpha_{i j} E_{i} \\
& -\frac{1}{6} \sum \beta_{i j k} E_{i} E_{j} E_{k}-\frac{1}{24} \sum \gamma_{i j k l} E_{i} E_{j} E_{k} E_{l}-\cdots,
\end{aligned}
$$

where $U(0)$ is the energy in the absence of the electric field $E, \mu_{i}=\left(\partial \mu_{i} / \partial E_{i}\right)_{U 0}, \alpha_{i j}=\left(\partial_{U i} / \partial E_{i} \partial_{E j}\right)_{U 0}, \beta_{i j k}=$ $\left(\partial_{U i} / \partial E_{i} \partial E_{j} \partial E_{k}\right)_{U 0}$, and $\gamma_{i j k l}=\left(\partial U i / \partial E_{j} \partial E_{k} \partial E_{l}\right)_{U 0}$. In the above equations the subscripts $i, j, k$, and $l$ refer to the molecular coordinate system and $E_{j}, E_{k}$, and so forth denote the components of the applied field. Note that these approximations are valid only for fields and polarizations that are small relative to atomic fields. When small electric fields are employed, the terms containing $\beta$ and $\gamma$ components can be ignored and the relation is assumed to be linear.

The values that are usually calculated are the molecular quantities: $\alpha, \beta$, and $\gamma$, which are the polarizability, firstorder hyperpolarizability, and the second-order hyperpolarizability tensors, respectively. A value that is useful in measuring second-order NLO properties is the mean first-order hyperpolarizability $\beta$, which is calculated as

$$
\beta_{\mathrm{tot}}=\left(\beta_{z x x}+\beta_{z y y}+\beta_{z z z}\right)
$$

The indices indicate the projection of field components in the direction indicated by the second two indices $\left(\beta_{z x x}\right)$ on the molecular axis indicated by the first index $\left(\beta_{z x x}\right)$, and the vectorial nature of $\beta$ for fixed molecular and field direction is clear. If we devise an experiment where the applied field is pointed in the direction of the molecular $x$-axis which is parallel to the dipolar axis of the molecule, $\beta_{x x x}$ will be the dominant contributor to the nonlinear response. Since the values of the first hyperpolarizability tensors of the output file of Hyperchem are reported in atomic units (a.u.), the calculated values were converted into electrostatic units ( 1 a.u. $=8.6393 \times$ $\left.10^{-33} \mathrm{esu}\right)$.
TABLE 1: Nonlinear optical properties for mono-O-Hydroxy Schiff base ligands 1-10.

\begin{tabular}{lcccc}
\hline Molecule & $\beta_{z x x}$ & $\beta_{z y y}$ & $\beta_{z z z}$ & $\begin{array}{c}\beta \\
\left(\times 10^{-30} \mathrm{esu}\right)\end{array}$ \\
\hline 1 & 204.219 & 852.595 & 1155.666 & 19.114 \\
2 & -240.801 & 1648.990 & 2784.197 & 36.219 \\
3 & -82.088 & 762.850 & 4224.402 & 42.377 \\
4 & 1341.625 & -1344.460 & 4442.795 & 38.358 \\
5 & 1890.550 & -31.837 & 16853.621 & 161.661 \\
6 & -7622.706 & 2416.968 & 14374.475 & 79.2114 \\
7 & -131.707 & 1555.308 & 2311.811 & 32.271 \\
8 & 622.288 & 924.831 & 1427.131 & 25.69545 \\
9 & -5168.761 & 1480.576 & 5752.509 & 17.834 \\
10 & -443.862 & 3076.680 & 4611.84959 & 62.588 \\
\hline
\end{tabular}

The calculated $\beta$ values have been converted into electrostatic units (esu) $\left(1\right.$ a.u. $=8.6393 \cdot 10^{-33}$ esu $)$.

$\beta_{\text {tot }}=\left(\beta_{z x x}+\beta_{z y y}+\beta_{z z z}\right)$.

\section{Results and Discussion}

The molecules studied in this investigation employing semiempirical AM1 calculation are shown in Figure 1. The study involves the calculation of first hyperpolarizability tensor for all 1-10 mono-O-Hydroxy Schiff bases ligands. It is intended to compare the electronic effect on the first hyperpolarizability of mono-O-Hydroxy Schiff bases ligands upon substitution of donor and acceptor groups at appropriate positions.

All first hyperpolarizabilities calculated for the molecules under investigation are given in Table 1 . The magnitude of the first hyperpolarizability tensor of all $\mathrm{N}$-salicylidene-aniline derivatives studied is dependent upon the availability of the lone pair of electrons on the nitrogen atom to conjugate with the N-salicylidene-aniline moiety.

The dramatic increase of first hyperpolarizability has been observed when the lone pair on the nitrogen atom of the donor group is forced to conjugate with the $\mathrm{N}$-salicylideneaniline ring system, upon substitution on nitrogen of $\mathrm{NH}_{2}$ group with other groups, such as methyl, ethyl, and methylpyrrolidine. It is expected that molecule 1 with $\mathrm{NH}_{2}$ as the donor group would give a higher value for first hyperpolarizability compared to molecule 7 where the donor group is $\mathrm{OCH}_{3}$. The enhancement of first hyperpolarizability from molecule 1 to 6 is attributed to the enhanced availability of the lone pair for conjugation with the $\mathrm{N}$-salicylidene-aniline system. The increase of $\beta$ for molecules $1-6$ is very significant.

Molecule 5 shows almost four times increase of first hyperpolarizability compared to molecule 4. Molecule 10 shows almost two times increase of first hyperpolarizability compared to molecule 7 . The significant difference between molecule 4 and molecule 5 is the introduction of an extra double bond, which helped to introduce two pyrrolidine rings to the system which will enhance the conjugation. The magnitude of the first hyperpolarizability tensor of molecule 5 is the largest relative to the other molecules. The molecular hyperpolarizability value of this molecule is about 1000 times that of urea $\left(0.14 \times 10^{-30} \mathrm{esu}\right)$. 
TABLE 2: HOMO, LUMO, and band gap energies $(\Delta E)$ for Schiff base ligands $1-10$.

\begin{tabular}{lccc}
\hline Molecule & $\begin{array}{c}\mathrm{HOMO} \\
(\mathrm{eV})\end{array}$ & $\begin{array}{c}\text { LUMO } \\
(\mathrm{eV})\end{array}$ & $\begin{array}{c}\Delta E(\mathrm{eV}) \\
\left(E_{\mathrm{LUMO}}-E_{\mathrm{HOMO}}\right)\end{array}$ \\
\hline 1 & -8.777 & -1.377 & 7.400 \\
2 & -8.567 & -1.325 & 7.242 \\
3 & -8.402 & -1.320 & 7.082 \\
4 & -8.686 & -1.485 & 7.201 \\
5 & -8.163 & -1.368 & 6.795 \\
6 & -8.548 & -1.854 & 6.694 \\
7 & -9.204 & -1.449 & 7.755 \\
8 & -8.614 & -0.932 & 7.682 \\
9 & -8.619 & -1.356 & 7.263 \\
10 & -8.772 & -1.878 & 6.894 \\
\hline
\end{tabular}

In molecules 8-10 consisting of combination of $\mathrm{NO}_{2}$ and $\mathrm{CN}$ as acceptor groups we observed a high value for $\beta$. The results obtained for molecule 9 when the R1 and R2 substituents were $-\mathrm{NH}_{2}$ and $-\mathrm{CN}$ groups, respectively, show a decrease for first hyperpolarizability compared to all molecules. This means that the presence of the $-\mathrm{NH}_{2}$ group together with the $-\mathrm{CN}$ in $\mathrm{N}$-salicylidene-aniline based ligands decreases the nonlinear optic property of these types of ligands.

The AM1 calculated HOMO-LUMO gaps for all Nsalicylidene-aniline derivatives are shown in Table 2 and summarized graphically in Figure 2 .

As shown in Table 2 substitution of different electron pushing groups on nitrogen of $\mathrm{NH}_{2}$ group in molecules 1-6 increases the energy of the molecular HOMO, while leaving the LUMO energy essentially unchanged due to the same acceptor group. Thus, the energy gap decreases with the substitution on nitrogen of $\mathrm{NH}_{2}$ and produces a larger hyperpolarizability.

The substitution of $-\mathrm{OCH}_{3}$ group for $\mathrm{NH}_{2}$ reduces the energy of the HOMO while leaving the LUMO energy unchanged. This has led to a larger energy gap than that of molecules 1-6 and produces a decrease in beta value. As can be seen from Figure 2 ligands 1-6 differ only by R1 substituent. The significant differences of the band gaps of these ligands indicate that the role of this substituent in prediction of the band gap is very important.

The substitution of $\mathrm{CN}$ group decreased the LUMO energy while keeping the HOMO unchanged which has led to a larger energy gap and shows a further reduction of the beta. In molecules 8-10 the HOMO is comparable but a large decrease in LUMO of molecule 10 has been observed and this has resulted in the smallest gap and the largest beta.

Figure 2 shows also the inverse relationship between calculated $\beta_{\text {tot }}$ and $E_{\text {gap }}$. The AM1 values for selected compounds show that it could be interesting to synthesize compounds with end parts in polyacetylene $\left(\mathrm{NO}_{2} / \mathrm{N}(\mathrm{Et})_{2}\right.$, $\mathrm{N}(\mathrm{Me})_{2}, \mathrm{NH}, \mathrm{NH}_{2}, \mathrm{NHNH}_{2}, \mathrm{NHOH}$, and $\mathrm{OH}$ ) groups having the greatest and the lowest, respectively, $\beta_{\text {tot }}$ and $E_{\text {gap }}$ values.

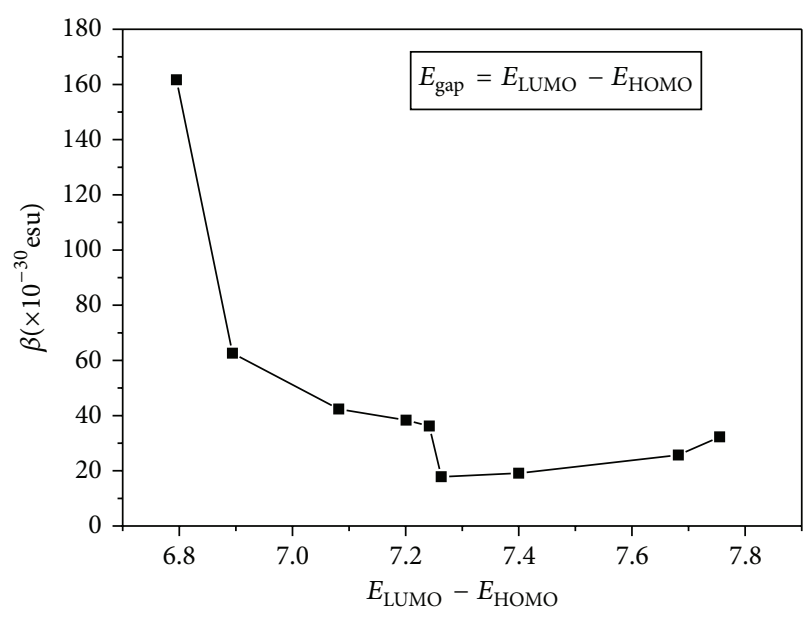

FIGURE 2: Variation of $\beta_{\text {tot }}$ and $E_{\text {gap }}$ values for Schiff base ligands.

The nonlinear optical (NLO) properties of conjugated molecules have been extensively studied as these compounds form a promising class of organic materials with interesting characteristics for photonic applications. Oudar and Zyss [15] found that the level of SHG response of a given material is inherently dependent upon its structural attributes. On a molecular scale, the extent of charge transfer (CT) across the NLO chromophore determines the level of SHG output. The greater the CT, the larger the SHG output. Chemla and Zyss [16] theoretical works have shown that the delocalization of $\Pi$-electrons in linear systems leads to large nonresonant optical molecular polarizabilities. Besides, geometric changes caused by incorporation of push-pull end groups can enhance the nonlinear polarizabilities of conjugated molecules.

Hayashi et al. [17] have calculated the linear and nonlinear polarizabilities in the side-chain direction of the polymer chains with all $\mathrm{H}$ atoms substituted by fluorine, hydroxyl, and cyano groups. Their results have shown that the coupling between electronic states of the side groups with those of the main chain increases the values of the perpendicular polarizabilities.

\section{Conclusions}

It is evident that the first hyperpolarizability $\beta$ tensor of $\mathrm{N}$ salicylidene-aniline derivatives strongly depends on the electronic structure of the molecule. In these molecules, where there is connectivity between two rings, system tends to rotate through the carbon-carbon sigma bond. This will increase the overlap of interacting orbitals, which will eventually increase the CT from donor to acceptor through the Nethylidenemethanamine moiety.

The HOMO-LUMO calculations show that the first hyperpolarizability of these derivatives is directly related to the HOMO-LUMO energy gap. The highest is molecule 5 which has the smallest energy gap while the smallest is 7 , which has the highest energy gap. The study reveals that these $\mathrm{N}$-salicylidene-aniline derivatives have important first static hyperpolarizabilities and may have potential applications 
in the development of NLO materials. It is important to stress that, in these calculated $\beta$ values, we do not take into account the effect of the field strength on the nuclear positions; we evaluate only the electronic component of $\beta$. The vibrational contributions which, for conjugated systems, can be important according to the NLO process are left for further investigations.

\section{Acknowledgment}

The author gratefully acknowledges the support of this work by the Research Laboratory of Chemical Materials, University of Batna in Algeria.

\section{References}

[1] A. Elmali, A. Karakaş, and H. Ünver, "Nonlinear optical properties of bis[(p-bromophenyl-salicylaldiminato)chloro]iron(III) and its ligand $\mathrm{N}$-(4-bromo)-salicylaldimine," Chemical Physics, vol. 309, no. 2-3, pp. 251-257, 2005.

[2] A. Karakaş, H. Ünver, A. Elmali, and I. Svoboda, "Study on the second order optical properties of $N$-(2, 4-dichloro)salicylaldimine," Zeitschrift für Naturforschung A, vol. 60, no. 5, pp. 376-382, 2005.

[3] J. F. Nicoud and R. J. Twieg, "Design and synthesis of organic molecular compounds for efficient second-harmonic generation," in Nonlinear Optical Properties of Organic Molecules and Crystals, D. S. Chemla and J. Zyss, Eds., vol. 1, pp. 227-296, Academic Press, New York, NY, USA, 1987.

[4] I. Ledoux, J. Zyss, F. Simoni, and C. Umeton, Novel Optical Materials and Applications, John Wiley \& Sons, New York, NY, USA, 1997.

[5] S. Di Bella, I. Fragala, I. Ledoux, M. A. Díaz-García, and T. J. Marks, "Synthesis, characterization, optical. Spectroscopy, electronic structure and second order nonlinear optical (NLO) properties of a novel class of donor-acceptor bis(salicylaldiminato)nickel(II) Schiff base NLO chromophores," Journal of the American Chemical Society, vol. 119, no. 40, pp. 9550-9557, 1997.

[6] G. Raos and M. del Zoppo, "Substituent effects on the secondorder hyperpolarisability of cyanine cations," Journal of Molecular Structure, vol. 589-590, pp. 439-445, 2002.

[7] A. Karakas, A. Elmali, H. Ünver, and I. Svoboda, "Nonlinear optical properties of some derivatives of salicylaldimine-based ligands," Journal of Molecular Structure, vol. 702, no. 1-2, pp.103110, 2004.

[8] M. Jalali-Heravi, A. A. Khandar, and I. Sheikshoaie, "Theoretical investigation of the structure, electronic properties and second-order nonlinearity of some azo Schiff base ligands and their monoanions," Spectrochimica Acta A, vol. 55, no. 12, pp. 2537-2544, 1999.

[9] P. N. Prasad and D. J. Williams, Introduction to Nonlinear Optical Effects in Molecules and Polymers, John Wiley \& Sons, New York, NY, USA, 1990.

[10] B. F. Levine and C. G. Bethea, "Second and third order hyperpolarizabilities of organic molecules," The Journal of Chemical Physics, vol. 63, no. 6, pp. 2666-2682, 1975.

[11] V. J. Docherty, D. Pugh, and J. O. Morley, "Calculation of the second-order electronic polarizabilities of some organic molecules. Part 1," Journal of the Chemical Society, Faraday Transactions II, vol. 81, no. 8, pp. 1179-1192, 1985.
[12] Hyper Chem, Molecular Modelling System, Hypercube Inc., Gainesville, Fla, USA, 2000.

[13] H. A. Kurtz, J. J. P. Stewart, and K. M. Dieter, "Calculation of the nonlinear optical properties of molecules," Journal of Computational Chemistry, vol. 11, no. 1, pp. 82-87, 1990.

[14] S. P. Karna and M. J. Dupuis, "Frequency dependent nonlinear optical properties of molecules: formulation and implementation in the HONDO program," Journal of Computational Chemistry, vol. 12, no. 4, pp. 487-504, 1991.

[15] J. L. Oudar and J. Zyss, "Relations between microscopic and macroscopic lowest-order optical nonlinearities of molecular crystals with one- or two-dimensional units," Physical Review A, vol. 26, no. 4, pp. 2028-2048, 1982.

[16] D. S. Chemla and J. Zyss, Nonlinear Optical Properties of Organic Molecules and Crystals, vol. 1, Academic Press, New York, NY, USA, 1987.

[17] S.-I. Hayashi, S. Yabushita, and A. Imamura, "Ab initio calculations of linear and nonlinear polarizabilities in the side-chain direction on the conjugated polymers," Chemical Physics Letters, vol. 179, no. 4, pp. 405-409, 1991. 

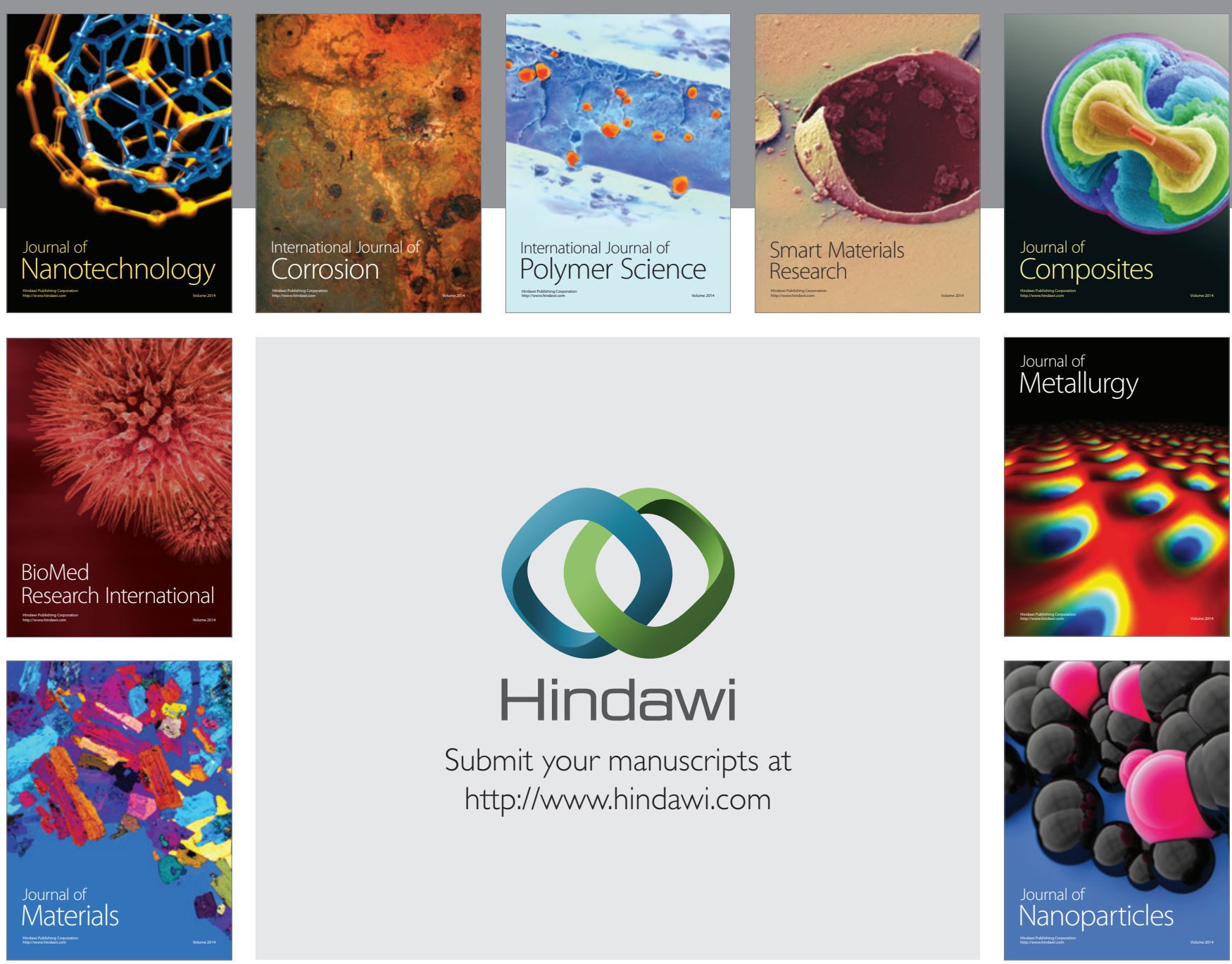

Submit your manuscripts at http://www.hindawi.com
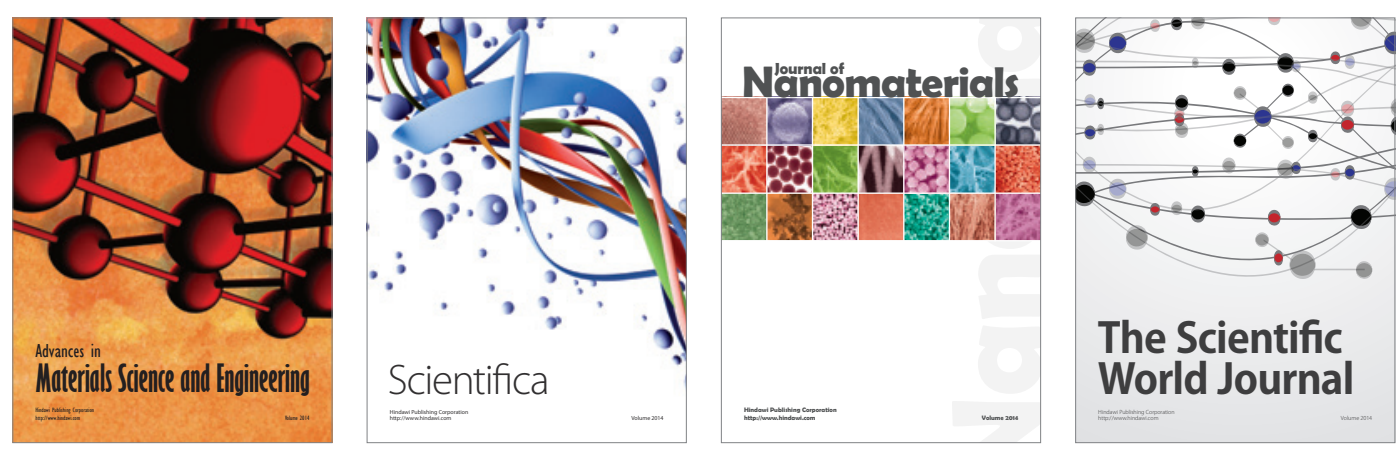

\section{The Scientific World Journal}
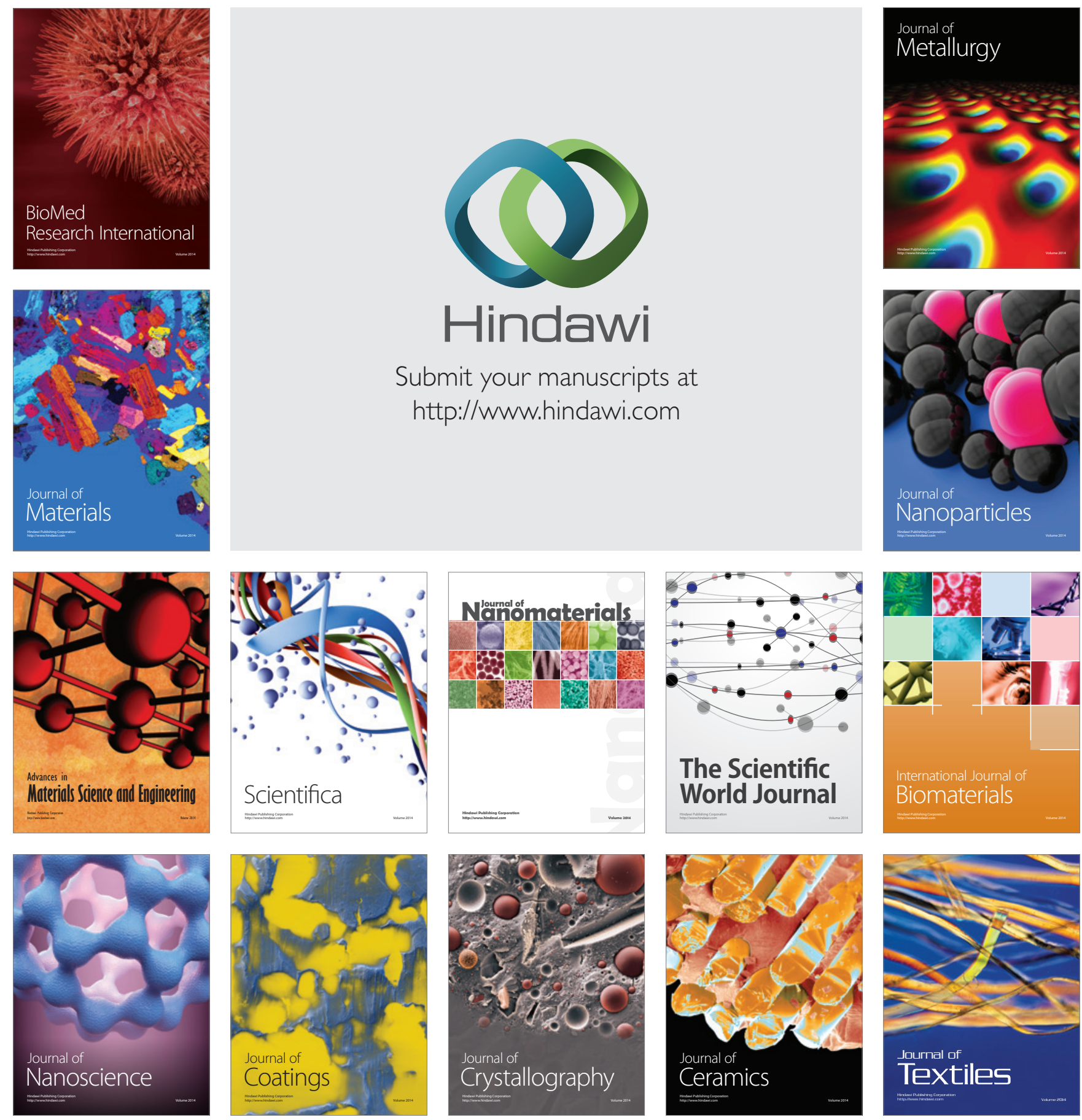